\title{
Clofarabine: a next-generation deoxyadenosine analogue
}

\begin{abstract}
Department of Medical
Services, Medical Advisor, Cadila Healthcare Limited, Zydus Tower, SG Highway, Ahmedabad, Gujarat, India

Received: 03 March 2018 Accepted: 03 April 2018

*Correspondence to:

Dr. Amit Bhalla,

Email: amitbhalla@

zyduscadila.com

Copyright: () the author(s), publisher and licensee Medip Academy. This is an openaccess article distributed under the terms of the Creative Commons Attribution NonCommercial License, which permits unrestricted noncommercial use, distribution, and reproduction in any medium, provided the original work is properly cited.
\end{abstract}

\author{
Amit Bhalla*
}

\begin{abstract}
Acute lymphoblastic leukaemia (ALL) is the most common of the paediatric leukaemias. It is estimated that the use of modern combination chemotherapy results in long-term remission in nearly $80 \%$ of children diagnosed with ALL. Despite therapy advances, approximately $20 \%$ of children with ALL, experience leukaemia relapse. Clofarabine (2-chloro-2'-fluoro-2'-deoxy-9- $\beta$-Darabinofuranosyladenine) is a second-generation nucleoside analogue and is structurally related to fludarabine and cladribine which are widely used in the treatment of lymphoproliferative disorders. Clofarabine exhibits greater affinity to deoxycytidine kinase (dCyd kinase) and prolonged retention in leukaemic blasts compared to fludarabine and cladribine. Clofarabine inhibits both DNA polymerases and ribonucleotide reductase (RNR). This results in impaired DNA synthesis through inhibition of DNA elongation as well as depletion of deoxyribonucleotides. Accumulation of clofarabine triphosphate, in the blasts of patients with refractory leukemia has been demonstrated. Prolonged intracellular half-life of 24 hours for clofarabine triphosphate. Clofarabine is indicated for the treatment of pediatric patients 1 to 21 years old with relapsed or refractory acute lymphoblastic leukemia after at least two prior regimens.
\end{abstract}

Keywords: Acute lymphoblastic leukaemia (ALL), Clofarabine

\section{INTRODUCTION}

Acute leukemia is the most common form of cancer in children, comprising approximately 30 percent of all childhood malignancies. The two most common types of acute leukemia in children are acute lymphoblastic leukemia (ALL) and acute myeloid leukemia (AML). ALL is characterised by malignant transformation of lymphoid progenitor cells in the bone marrow, blood and extramedullary sites, and is the most common of the paediatric leukaemias. It occurs about 5 times more commonly than does AML.

Studies from India have reported that ALL accounted for 60 to $85 \%$ of all childhood leukemias. ${ }^{1-3}$
The proportion of children with acute lymphoblastic leukaemia (ALL) successfully cured with chemotherapy has progressively increased over time. It is estimated that the use of modern combination chemotherapy results in long-term remission in nearly $80 \%$ of children diagnosed with ALL.

Despite these advances, approximately $20 \%$ of children with ALL experience leukaemia relapse, which remains the leading cause of treatment failure (Pui and Evans, 2006). While a substantial proportion of children with relapsed ALL achieve a second remission, the final outcome of paediatric patients who experience leukaemia recurrence remains unsatisfactory. 


\section{Clofarabine}

Clofarabine (2-chloro-2'-fluoro-2'-deoxy-9- $\beta$-Darabinofuranosyladenine) is a second-generation nucleoside analogue and is structurally related to fludarabine and cladribine which are widely used in the treatment of lymphoproliferative disorders.

As shown in Figure 1, the drug has been rationally designed in an attempt to combine the therapeutic qualities and overcome the toxic limitations, especially doselimiting neurotoxicity, of its congeners.

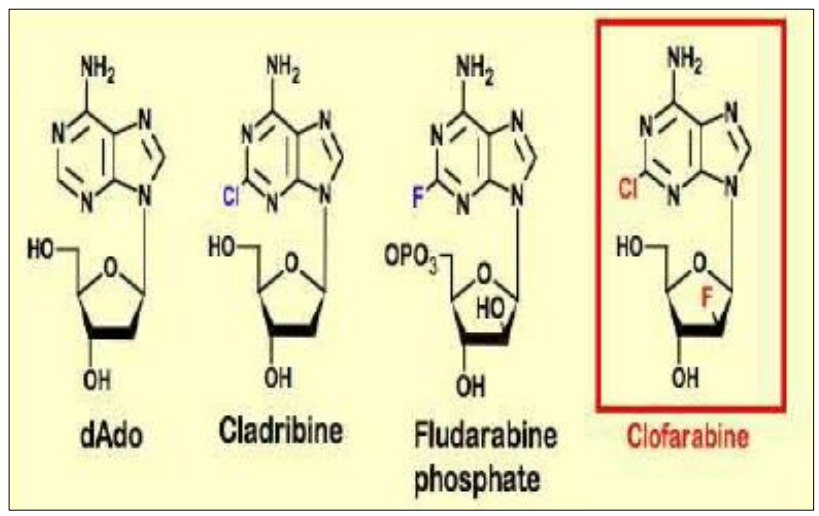

Figure 1: Chemical structure of Clofarabine and its congeners. ${ }^{4}$

Although developed in the 1980's, there has been little interest in its development until 1993 when the first preclinical and animal studies were initiated at MD Anderson Cancer Center. Human phase I trials took off in 1998 and the drug was approved by the Food and Drug Administration (FDA) in 2004 for treatment of relapsed and refractory ALL in children and adolescents up to the age of 21 years.

Approval by the European Medicines Agency (EMA) followed in 2006.

This section reviews the pharmacology, toxicities and clinical efficacy of clofarabine.

\section{PHARMACODYNAMICS}

Like other purine analogs, clofarabine retains the 2halogenated aglycone, which renders these compounds resistant to deamination by adenosine deaminase. In addition, clofarabine contains a second halogen atom, fluorine, at the 2'-position of the carbohydrate (Figure 1), which inhibits glycosidic linkage in order to protect it from bacterial purine nucleoside phosphorylase and from acid hydolysis. This chemical configuration prevents release of neurotoxic halogenated adenine, which is the reason for the dose-limiting neurotoxic side effects of similar nucleoside analogs. ${ }^{5}$ The stability of clofarabine in an acidic environment makes for a potential orally available anticancer drug, and there is ongoing research in this area.

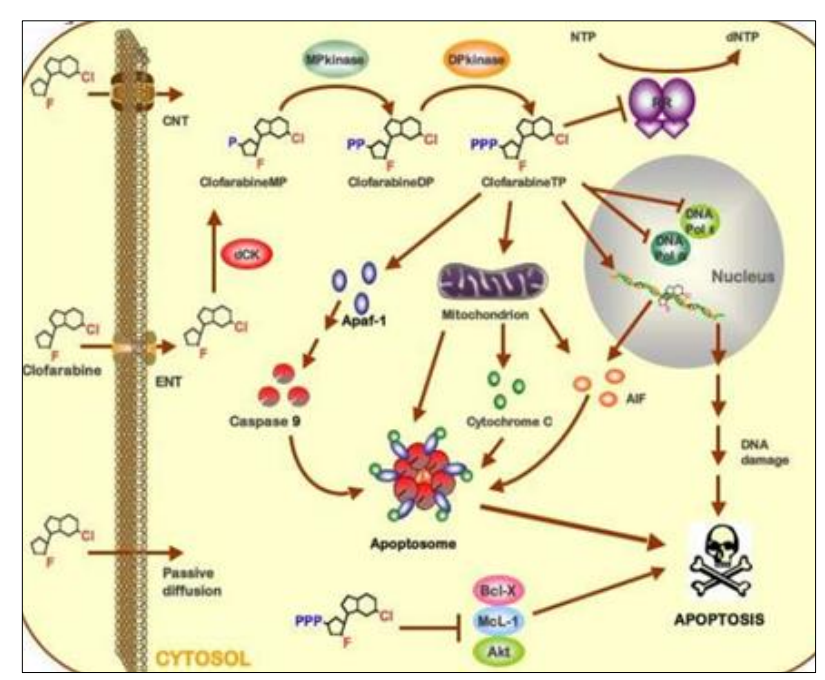

Figure 2: Clofarabine mechanism of action. ${ }^{4}$

Similar to other nucleoside analogues, clofarabine requires intracellular phosphorylation to its triphosphate form by deoxycytidine kinase (dCyd kinase) to become active (Figure 2).

Clofarabine exhibits greater affinity to dCyd kinase and prolonged retention in leukaemic blasts compared to fludarabine and cladribine. While fludarabine preferably inhibits DNA polymerases, cladribine is primarily an inhibitor of ribonucleotide reductase (RNR). Clofarabine inhibits both DNA polymerases and RNR. This results in impaired DNA synthesis through inhibition of DNA elongation as well as depletion of deoxyribonucleotides.

This mechanism leads to depletion of the intracellular deoxynucleotide triphosphate pool, as a result of which clofarabine self-potentiates the incorporation of clofarabine triphosphate into DNA, thereby amplifying the effectiveness of DNA synthesis inhibition.

Furthermore, clofarabine shows activity in non-dividing cells and cells with a low proliferation rate, probably through disruption of mitochondria with release of proapoptotic mitochondrial factors (such as mitochondrial cytochrome C) caused by damage of the integrity of the mitochondrial membrane. Induction of the apoptotic pathway leads to programmed cell death, even in nonproliferating human lymphocytes, and could therefore be in part the explanation for the enhanced cytotoxic activity of clofarabine.

\section{PHARMACOKINETICS}

Clofarabine is $47 \%$ bound to plasma protein. Accumulation of clofarabine triphosphate, in the blasts of patients with refractory leukemia has been demonstrated. Prolonged intracellular half-life of 24 hours for clofarabine triphosphate. The terminal half-life of clofarabine is estimated to be 5.2 hours. 


\section{RATIONALE FOR CLOFARABINE COMBINATIONS}

As clofarabine is a potent inhibitor of ribonucleotidereductase $(\mathrm{RnR})$, it was hypothesized that clofarabine would accumulate cytarabine triphosphate in leukemic cells and increase the antileukemic activity of cytarabine. When clofarabine inhibits $\mathrm{RnR}$, it will result in decrease of deoxynucleotide triphosphate and it subsequently cause inhibition of dCK by a feedback mechanism. Therefore, a combination of clofarabine and cytarabine increases retention of ara-CTP.

Clofarabine is an inhibitor of DNA synthesis as well as repair. For this reason, several combinations of clofarabine and DNA-damaging agents such as cyclophosphamide, epipodophyllotoxins and anthracyclines have been explored.

\section{Clinical studies in Acute lymphoblastic leukaemia (Children and adolescents)}

The maximum tolerated dose (MTD) of clofarabine for paediatric patients with relapsed and refractory acute leukaemia has been determined to be $52 \mathrm{mg} / \mathrm{m}^{2}$ intravenously per day for 5 days. ${ }^{6}$ Dose-limiting toxicities (DLTs) occurred at $70 \mathrm{mg} / \mathrm{m}^{2}$. One patient had grade 4 hyperbilirubinaemia and grade 3 elevation of transaminases, another patient experienced grade 3 skin rash. A complete remission rate (CR) of $24 \%$ was observed in 17 heavily pretreated ALL patients. One third of patients had undergone prior stem cell transplantation. The remissions achieved with clofarabine were durable enough to allow several patients to proceed to stem cell transplantation. In 2002, a multicenter phase II study started enrolling pediatric patients with relapsed or refractory ALL. ${ }^{7}$ Median age was 12 years (range, 1 to 20 years). One third of the 61 patients relapsed after earlier stem cell transplantation and the median number of previous induction regimens was 3 . An overall response rate (ORR) of $30 \%$ [20\% CR/CR with incomplete platelet recovery (CRp) and $10 \%$ partial remission (PR)] was achieved with a median of 2 cycles of clofarabine at $52 \mathrm{mg} / \mathrm{m}^{2} /$ day for 5 days. These results led to FDA approval of clofarabine for patients aged 1-21 years who suffer from relapsed or refractory ALL after at least two preceding regimens. One potential strategy to improve on these results is to combine clofarabine with other chemotherapeutic drugs. The most extensively studied drug combination in paediatric ALL is clofarabine with cyclophosphamide and etoposide. The rationale for this combination is that pretreatment with clofarabine might hamper repair of cyclophosphamide and etoposide induced DNA-damage and lead to increased apoptotic cell death. A phase I study determined the MTDs of clofarabine, cyclophosphamide and etoposide to be $40 \mathrm{mg} / \mathrm{m}^{2}$, $440 \mathrm{mg} / \mathrm{m}^{2}$ and $100 \mathrm{mg} / \mathrm{m}^{2}$ respectively, each daily for five consecutive days. An ORR of 55\% was observed in this study. ${ }^{8}$ Locatelli et al, explored a slightly different dosage scheme. Twenty-five patients with refractory or multiply relapsed ALL received clofarabine $40 \mathrm{mg} / \mathrm{m}^{2}$, cyclophosphamide $400 \mathrm{mg} / \mathrm{m}^{2}$ and etoposide $150 \mathrm{mg} / \mathrm{m}^{2}$ for 5 days. ${ }^{9}$ This combination yielded a CR rate of $54 \%$ and permitted half of the responding patients to proceed to stem cell transplantation. While $76 \%$ of B-ALL patients $(n=17)$ responded, the response rate in T-ALL $(n=8)$ was only $12 \%$. This combination regimen was well-tolerated with reversible liver function abnormalities, profound myelosuppression and febrile neutropaenia being the most common toxicities. There were no treatment-related deaths.

To investigate its value in the frontline treatment of ALL Escherich et al, applied clofarabine $5 \times 40 \mathrm{mg} / \mathrm{m}^{2}$ in combination with pegylated asparaginase (PEG-ASP) $1 \times 2500 \mathrm{iu} / \mathrm{m}^{2}$ in high risk ALL patients as a novel postinduction element in the German Co-operative Study Group for treatment of ALL (CoALL) trial 08-09. ${ }^{10}$ Newly diagnosed ALL patients, defined by a significant minimal residual disease (MRD) load at the end of induction (Bprogenitor ALL at day $29 \geq 10-4$ and T-ALL at day $43 \geq 10$ 3 ) were eligible for this phase II trial. All other patients received the standard treatment consisting of high-dose cytarabine (HIDAC) $4 \times 3 \mathrm{~g} / \mathrm{m}^{2}$ in combination with PegASP $2500 \mathrm{iu} / \mathrm{m}^{2}$. Forty-two patients (39 B-progenitor; $3 \mathrm{~T}$ ALL) fulfilled the criteria, were stratified and received the clofarabine/PEG-ASP treatment resulting in 24/39 (61\%) MRD-negative B-progenitor patients compared to $18 / 39$ (46\%) after HIDAC/PEG-ASP in CoALL 07-03. Overall there was a strong response to the treatment with clofarabine/PEG-ASP measured by MRD. Twenty-four out of $39(61 \%)$ B-progenitor patients were MRD-negative after clofarabine/PEG-ASP treatment.

\section{Safety}

The most common toxicities observed during exposure to Clofarabine were gastrointestinal system adverse events (AEs) (including vomiting, nausea, and diarrhea), adverse hematologic effects (including anemia, leukopenia, thrombocytopenia, neutropenia, and febrile neutropenia), and infection. It should be noted that a large number of the adverse effects were already present in many of the patients at baseline. Other significant groups of AEs observed for Clofarabine are found in the hepatobiliary, renal, and gastrointestinal organ systems. In addition, tumor lysis syndrome, capillary leak syndrome or SIRS have been reported.

The following serious adverse reactions have been reported with Clofarabine:

- Enterocolitis

- Severe bone marrow suppression

- Hemorrhage

- Hepatotoxicity

- Hyperuricemia (Tumor Lysis Syndrome)

- $\quad$ Systemic Inflammatory Response Syndrome (SIRS) and Capillary Leak Syndrome

- Infections, including severe sepsis 
- $\quad$ Stevens-Johnson syndrome (SJS)

\section{Dosing schedule and administration}

Clofarabine is indicated for the treatment of pediatric patients 1 to 21 years old with relapsed or refractory acute lymphoblastic leukemia after at least two prior regimens. This use is based on the induction of complete responses. Approval is based on objective response rates.

- The pediatric (ages 1-21) dose of $52 \mathrm{mg} / \mathrm{m}^{2}$ of body surface area is administered by intravenous infusion over 2 hours daily for 5 consecutive days. Longer infusion times should be considered in children weighing $<20 \mathrm{~kg}$.

- Treatment cycle comprises a 5-day course of clofarabine followed by the recovery period of 2 to 6 weeks (median 4 weeks) from the starting day of the previous cycle.

- The cycles are repeated following recovery or return to baseline organ function. In clinical trials in pediatric acute leukemias, the median duration of a cycle was 28 days (range of 12-55 days).

\section{CONCLUSION}

Clofarabine is a novel, next-generation, halogenatedadenosine analogue.

Clofarabine has several distinguishing features compared to other drugs in its class including.

1. Greater resistance to cellular degradation by adenosine deaminase,

2. Increased stability in gastric acid, decreased susceptibility to phosphorolytic cleavage,

3. More efficiency as a substrate for deoxycytidine kinase (exceeding cladribine and the natural substrate deoxycytidine),

4. Unlike fludarabine, clofarabine is active in nondividing cells and in cells with a low proliferation rate by inducing apoptosis through its direct and indirect action on mitochondrial function.

Clofarabine is indicated for the treatment of acute lymphoblastic leukaemia (ALL) in paediatric patients who have relapsed or are refractory after receiving at least two prior regimens.

Funding: No funding sources Conflict of interest: None declared

Ethical approval: Not required

\section{REFERENCES}

1. Biswas S, Chakrabarti S, Chakraborty J, Paul PC, Konar A, Das S. Childhood acute leukemia in West Bengal, India with an emphasis on uncommon clinical features. Asian Pac J Cancer Prev. 2009;10:903-6.

2. Swaminathan R, Rama R, Shanta V. Childhood cancers in Chennai, India, 1990-2001: Incidence and survival. Int J Cancer. 2008;122:2607-11.

3. Arora RS, Eden TO, Kapoor G. Epidemiology of childhood cancer in India. Indian $\mathrm{J}$ Cancer. 2009;46:264-73.

4. Zhenchuk A, Lotfi K, Juliusson G, Albertioni F. Mechanisms of anti-cancer action and pharmacology of clofarabine. Biochemical pharmacology. 2009 Dec 1;78(11):1351-9.

5. Cheson BD, Vena DA, Foss FM, Sorensen JM. Neurotoxicity of purine analogs: A review. J Clin Oncol. 1994;12(10):2216-28.

6. Jeha S, Gandhi V, Chan KW, McDonald L, Ramirez I, Madden R, et al. Clofarabine, a novel nucleoside analog, is active in pediatric patients with advanced leukemia. Blood. 2004 Feb 1;103(3):784-9.

7. Kantarjian HM, Gandhi V, Kozuch P, Faderl S, Giles F, Cortes J, et al. Phase I clinical and pharmacology study of clofarabine in patients with solid and hematologic cancers. Journal of Clinical Oncology. 2003 Mar 15;21(6):1167-73.

8. Hijiya N, Gaynon P, Barry E, Silverman L, Thomson $\mathrm{B}$, Chu R, et al. A multi-center phase I study of clofarabine, etoposide and cyclophosphamide in combination in pediatric patients with refractory or relapsed acute leukemia. Leukemia. 2009 Dec;23(12):2259.

9. Locatelli F, Testi AM, Bernardo ME, Rizzari C, Bertaina A, Merli $\mathrm{P}$, et al. Clofarabine, cyclophosphamide and etoposide as single-course reinduction therapy for children with refractory/multiple relapsed acute lymphoblastic leukaemia. British Journal of haematology. 2009 Nov 1;147(3):371-8.

10. Escherich G, Stadt U, Zimmermann M, Horstmann MA. Clofarabine in combination with pegylated asparaginase in the frontline treatment of childhood acute lymphoblastic leukaemia: a feasibility report from the CoALL 08-09 trial. British Journal of Haematology. 2013 Oct 1;163(2):240-7.

Cite this article as: Bhalla A. Clofarabine: a nextgeneration deoxyadenosine analogue. Int J Basic Clin Pharmacol 2018;7:1048-51. 